\title{
APS physics
}

This is the accepted manuscript made available via CHORUS. The article has been published as:

Complete photonic band gaps in supercell photonic crystals Alexander Cerjan and Shanhui Fan

Phys. Rev. A 96, 051802 - Published 13 November 2017

DOI: 10.1103/PhysRevA.96.051802 


\title{
Complete photonic bandgaps in supercell photonic crystals
}

\author{
Alexander Cerjan and Shanhui Fan \\ Department of Electrical Engineering, and Ginzton Laboratory, \\ Stanford University, Stanford, California 94305, USA
}

\begin{abstract}
We develop a class of supercell photonic crystals supporting complete photonic bandgaps based on breaking spatial symmetries of the underlying primitive photonic crystal. One member of this class based on a two-dimensional honeycomb structure supports a complete bandgap for an index-contrast ratio as low as $n_{\text {high }} / n_{\text {low }}=2.1$, the lowest index constrast known to support a complete bandgap for a $2 \mathrm{D}$ photonic crystal. This same design principle is used to develop the first photonic crystal slab that supports a bandgap for dual-polarization visible light. The complete bandgaps found in such supercell photonic crystals do not necessarily monotonically increase as the index-contrast in the system is increased.
\end{abstract}

Since their discovery, photonic crystals have become an indispensable technology across the entire field of optical physics due to their ability to confine and control light of an arbitrary wavelength [1-4]. This critical feature is achieved by designing the crystal lattice to possess a complete photonic bandgap, a range of frequencies for which no light can propagate regardless of its momentum or polarization. Unlike their electronic counterparts in conventional crystals, whose band structure is limited to the crystal lattices available in atomic and molecular structures, the dielectric structure comprising a photonic crystal can be specified with nearly complete arbitrariness, yielding a vast design space for optimizing photonic crystals for specific applications that is limited only by the index of refraction of available materials at the operational wavelength. For example, photonic crystals have been developed to enable complete absorption in monolayer materials [5, 6], or for use in achieving high-power solid-state lasers [7-9]. Moreover, this design freedom in dielectric structures has been leveraged in numerous studies to optimize the complete bandgaps in high-index materials [10-33]. Similar efforts to realize new crystal structures or improve upon existing ones to achieve complete bandgaps in low-index materials have yielded only minor improvements upon traditional simple crystal structures with high symmetry $[34,35]$, i.e. the inverse triangular lattice in two dimensions (2D) [4] and the network diamond lattice in three dimensions (3D) [36], or have been obtained directly through numerical simulation $[30,33]$.

However, the ability to realize complete bandgaps for low-index materials is critically important to the development of many photonics technologies operating in the visible wavelength range, such as augmented and virtual reality systems, where the highest-index lossless materials have $n \approx 2.3-2.5$ [37-39]. Currently, there are no known photonic crystal slabs which realize dualpolarization in-plane complete bandgaps using these materials. Although a few $3 \mathrm{D}$ photonic crystals do display a complete bandgap in this range, 3D photonic crystals are difficult to fabricate [40-44].

Here, we demonstrate a new class of complete photonic bandgaps which are achieved by judiciously break- ing symmetry, rather than promoting it. By starting with a photonic crystal possessing a large bandgap for one polarization, we show that by expanding the primitive cell of the photonic crystal to form a supercell and then slightly adjusting the dielectric structure within this supercell to break part of the translational symmetry with respect to the original primitive cell, a bandgap in the other polarization can be opened, thus producing a complete bandgap. This method yields a twodimensional photonic crystal based on a honeycomb lattice with a complete bandgap that persists down to an index-contrast ratio of $n_{\text {high }} / n_{\text {low }}=2.1$, the lowest known index-contrast ratio in $2 \mathrm{D}$ photonic crystals. Such low index contrast bandgaps can also be translated into photonic crystal slabs. In contrast to the complete photonic bandgaps found in traditional photonic crystals, complete bandgaps in supercell photonic crystals do not necessarily monotonically increase as a function of the index-contrast ratio, disproving a generally held intuition in the photonic crystal literature [4].

To illuminate how symmetry breaking can help to realize complete photonic bandgaps, we first consider the 2D photonic crystal comprised of a network structure on a honeycomb lattice depicted in Fig. 1(a). The primitive cell of this system contains a pair of vertices in this network lattice, and the system can be parameterized solely in terms of the thickness, $t$, of the lines forming the network structure. Although in a low-index network structure, $n_{\text {high }} / n_{\text {low }}=2.4$, a wide range of $t$ yields a large transverse electric (TE) bandgap as shown in Fig. 1(b), no complete photonic bandgap exists for any choice of $t$ for this choice of $n_{\text {high }} / n_{\text {low }}$.

However, starting from the crystal structure as shown in Fig. 1(a), we can find a complete bandgap in a closely related supercell photonic crystal. First, we increase the size of the primitive cell to contain six vertices which form the supercell, as depicted in Fig. 1(c). In doing so, each of the bands in the primitive Brillouin zone fold up into three bands in the supercell Brillouin zone, shown in Fig. 1(d). Along the edge of the supercell Brillouin zone $\left(M^{\prime} \rightarrow K^{\prime}\right)$, pairs of the folded supercell bands can form lines of degeneracies, i.e. degenerate contours [46], and one such degenerate contour is formed per trio 

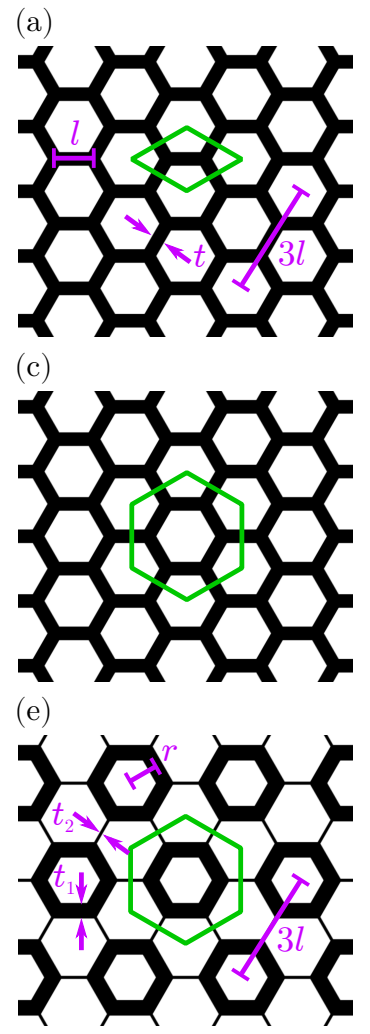

(b)

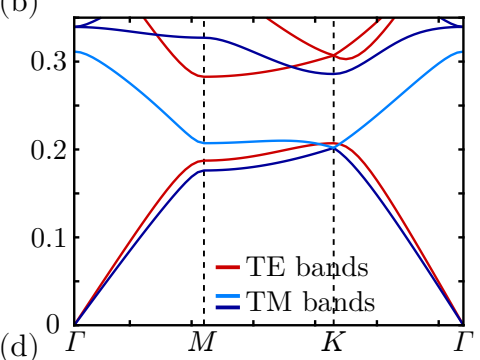

\section{(d)}

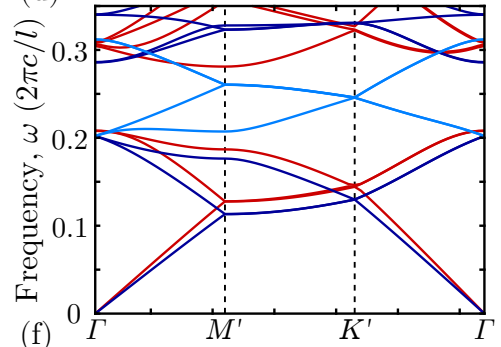

(f)

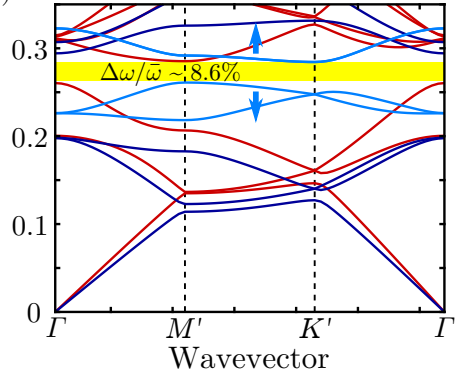

FIG. 1. (a),(c),(e) Schematics of 2D photonic crystals formed of a network of a high dielectric material with $n=2.4$ (black region) in a background of air, $n_{\text {air }}=1$. The unit cell for the corresponding band structure in (b),(d),(f) is shown as a green diamond or hexagon. Parameters which characterize these crystals are indicated in purple. The structures in (a) and (c) are completely specified by the line thickness, $t$, while the structure in (e) is completely specified by three parameters, the thick line thickness, $t_{1}$, the thin line thickness, $t_{2}$, and the shortest distance from a thick line to the center of its corresponding thick-bordered hexagon, $r$. $l$ denotes the distance between two vertices in the primitive honeycomb lattice. (b),(d),(f) Band structures around the border of the irreducible Brillouin Zone for the photonic crystals shown in (a),(c),(e), respectively, in which the TE bands are shown in red and the TM bands are shown in blue. In (b), the $2^{\text {nd }} \mathrm{TM}$ band is shown as cyan (light gray), while the corresponding folded bands in the supercell Brillouin zone in (d) and (f) are also shown in the same color. In (b), (d), $t / l=0.3636$, while in (f), $t_{1} / l=0.4149, t_{2} / l=0.0816$, and $r / l=0.8145$, and a complete bandgap is found with width $\Delta \omega / \bar{\omega}=8.6 \%$ between the $8^{\text {th }}$ and $9^{\text {th }}$ bands (yellow rectangle). Band structures were calculated using MIT Photonic BANDS (MPB) [45].

of folded bands originating from the same band in the primitive Brillouin zone. From the perspective of the supercell photonic crystal, the degeneracies comprising each of the degenerate contours are accidental, and are only the result of the supercell obeying an extra set of spatial symmetries as it is a three-fold copy of the original

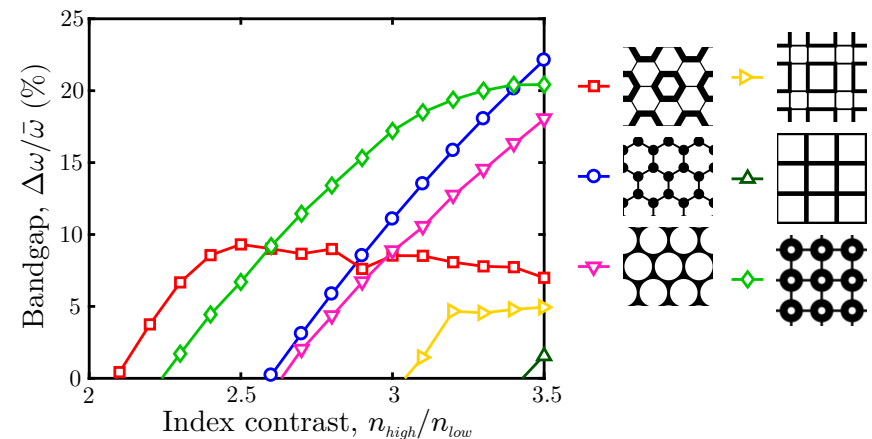

FIG. 2. Plot of the optimized complete bandgap width as a function of the index contrast, $n_{\text {high }} / n_{\text {low }}$ for six different 2D photonic crystals, the supercell network honeycomb lattice discussed in Fig. 1 (red squares), the decorated square lattice designed in Ref. [30] (green diamonds), the traditional inverse triangular lattice (magenta downward-pointing triangles), the decorated network honeycomb lattice designed in Ref. [34] (blue circles), the traditional network square lattice (dark green upward-pointing triangles), and the supercell network square lattice discussed in Fig. 4 (yellow right-pointing triangles). In the schematics, the black regions correspond to the high index material, $n_{h i g h}$, while the white regions correspond to low index material, $n_{\text {low }}$. Optimized complete bandgaps were calculated using MPB [45].

primitive cell. Thus by breaking these symmetries, the degeneracies forming the degenerate contours are lifted, and a gap can begin to open between the two transverse magnetic (TM) bands. The supercell is now characterized in terms of three parameters, the thickness of the center lines, $t_{1}$, the thickness of the connecting lines, $t_{2}$, and the size of the thick-lined hexagons, $r$, shown in Fig. $1(\mathrm{e})$. When the symmetry breaking becomes sufficiently strong, a complete photonic bandgap opens between the 8th and 9th bands of the system, whose maximum gap width at $n_{\text {high }} / n_{\text {low }}=2.4$ can be found numerically to be $\Delta \omega / \bar{\omega}=8.6 \%$. Here, $\Delta \omega$ is the difference between the minimum of the 9 th band and the maximum of the 8 th band, while $\bar{\omega}$ is the central frequency of the gap. Rigorously, after the symmetry of this system is broken, the supercell containing six vertices becomes the primitive cell of the system. However, for semantic convenience, we will continue to refer to this larger primitive cell as the 'supercell' and reserve 'primitive cell' for the smaller system whose symmetry is intact.

Previously, the lowest index-contrast ratio known to support a complete bandgap in a $2 \mathrm{D}$ photonic crystal was a decorated square lattice, shown as the green curve in Fig. 2, which has a complete bandgap between the 5 th and 6 th bands for index-contrast ratios as low as $n_{\text {high }} / n_{\text {low }}=2.3[30]$. In contrast, the supercell honeycomb lattice possesses a complete bandgap for indexcontrast ratios as low as $n_{\text {high }} / n_{\text {low }}=2.1$, and as such is the first $2 \mathrm{D}$ photonic crystal design that can realize a significant complete bandgap for visible wavelengths in thin film Titanium Dioxide, $n=2.3-2.4$ [47]. Furthermore, 
(a)

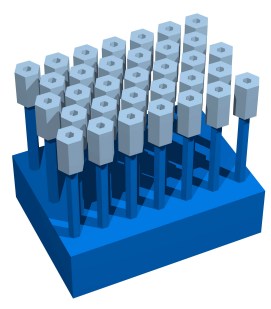

(b)

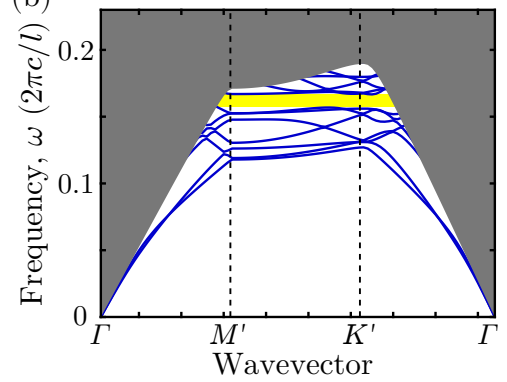

FIG. 3. (a) Schematics of a 3D photonic crystal slab consisting of Titanium Dioxide hexagonal rods with $n=2.57$ at $\lambda=650 \mathrm{~nm}$ mounted on a periodic substrate of silica, $n=1.45$, with air above, $n=1$. Each Titanium Dioxide hexagonal rod also contains a hexagonal air hole in its center, while the substrate rods are solid. This system can be completely parameterized using exactly the same choice of $t_{1}, t_{2}$, and $r$ from the 2D photonic crystal shown in Fig. 1(e), as well as the height of each rod, $h$, and the width of the substrate rods, $w$. For the structure in (a) $t_{2}=0$. (b) Band diagram for the photonic crystal slab shown in (a). The grey regions of the band diagram indicate the continuum of radiation modes which lies above the light line. As can be seen, a complete bandgap with width $\Delta \omega / \bar{\omega}=5.6 \%$ (yellow rectangle) opens between the $7^{\text {th }}$ and $8^{\text {th }}$ bands for $t_{1} / l=0.7482, t_{2} / l=0$, $r / l=0.7405, h / l=3.750$, and $w / l=1.0392$, where $l$ is again the distance between two vertices in the underlying primitive honeycomb lattice. For $\bar{\lambda}=650 \mathrm{~nm}$ tuned to the center of the bandgap, the bandgap would extend over the range 633$667 \mathrm{~nm}$, with $t_{1}=78.9 \mathrm{~nm}, r=78.0 \mathrm{~nm}, h=394.9 \mathrm{~nm}$, and $w=109.4 \mathrm{~nm}$. Likewise, for $\bar{\lambda}=1.55 \mu \mathrm{m}$, the bandgap extends over $1.509-1.591 \mu \mathrm{m}$, with $t_{1}=188 \mathrm{~nm}, r=186 \mathrm{~nm}$, $h=941 \mathrm{~nm}$, and $w=261 \mathrm{~nm}$. Band structures were calculated using MPB [45].

this supercell honeycomb structure could also be used in conjunction with high-index materials available in other frequency ranges so that the low-index material used in the structure need not be air. For example, optical fibers with silicon cores in silica cladding [48], as well as silicon photonic crystal fibers in air [49] have been recently fabricated. Thus, the photonic crystal design presented here could realize complete bandgaps at the on-axis wavevector $k_{\|}=0$ in completely solid photonic crystal fibers operating in the communications band, where the high index regions are silicon, $n_{\text {high }}=3.48$, and the low-index regions are filled with fused silica, $n_{\text {low }}=1.45$, such that $n_{\text {high }} / n_{\text {low }}=2.4$ for $\lambda=1.55 \mu \mathrm{m}$. This is in contrast to traditional silica-air based photonic crystal fibers [5053], which support a complete bandgap only at a large on-axis wavevector, $k_{\|} \neq 0$. Photonic crystal fibers operating near $k_{\|}=0$ can be used to enhance nonlinear interactions, such as phase sensitivity [54], Raman scattering $[55,56]$, and fiber sensors [57-60].

The same design principle can also be used to develop new photonic crystal slabs so as to provide confinement in three dimensions. In Fig. 3, we show a supercell honeycomb slab with a complete below-light-line

dual-polarization bandgap of $\Delta \omega / \bar{\omega}=5.6 \%$ for Titanium Dioxide on a periodic silica substrate. Note that in photonic crystal slabs, to define a bandgap one only considers the phase space regions below the light line, as above the light line the radiations modes form a continuum with no gaps. This system with the center of the bandgap tuned to $\bar{\lambda}=650 \mathrm{~nm}$ possesses a complete bandgap between $633 \mathrm{~nm}$ and $667 \mathrm{~nm}$, and has a minimum feature size of $78 \mathrm{~nm}$, which is well above the resolution limits of modern lithography techniques $[61,62]$. This represents the first realistic design of a photonic crystal slab system with a complete polarization-independent bandgap in the visible wavelength range. This design can also be realized using diamond as the high-index material [63-65]. Moreover, as the bandgap in this slab system is for both polarizations, it persists even when the substrate is introduced. In contrast, single-polarization bandgaps in slab systems disappear with the introduction of a substrate, as it breaks the mirror symmetry in $z$ and couples the two polarizations together [66]. Finally, this design could also be used to realize entirely solid photonic crystal slabs for communications frequencies where higher index dielectric materials are available.

The procedure used above is not restricted to the honeycomb lattice. To illustrate this point, we use the same method to produce a complete bandgap in a $2 \mathrm{D}$ supercell square network lattice, as shown in Fig. 4. Unlike in the primitive honeycomb crystal, the TE bandgap in the underlying primitive square lattice is spanned by two TM bands. Thus, a complete bandgap is only realized for sufficiently strong symmetry breaking so that not only does a gap open in each degenerate contour of the folded supercell TM bands, but that a gap opens between these two folded bands. This limits the overall width of the complete bandgap, and the lowest index-contrast ratio for which this structure possesses a complete bandgap is $n_{\text {high }} / n_{\text {low }} \sim 3.1$, as shown as the yellow line in Fig. 2 .

Complete bandgaps in supercell photonic crystal possess two features which distinguish them from complete bandgaps found in traditional photonic crystals. First, as noted above, these structures have been designed by specifically breaking symmetry within the system. This is entirely distinct from what is observed for bandgaps found in traditionally designed structures, which consider the high-symmetry triangular lattice in $2 \mathrm{D}$ and diamond lattice in 3D to be near optimal. Second, as can be seen in Fig. 2, complete bandgaps in supercell structures do not necessarily monotonically increase in size as a function of the index-contrast. This example disproves the generally held intuition that the bandgap (between the same two bands) of an optimized structure increases or stays constant as the index contrast increases. However, related structures featuring additional degrees of freedom beyond the three shown in Fig. 1(e) possess complete bandgaps which are constant as the index-contrast is increased, see Appendix A.

Designing two-dimensional supercell photonic crystals to possess complete bandgaps has three steps. First, a 
(a)

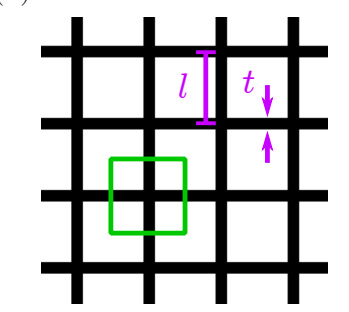

(c)

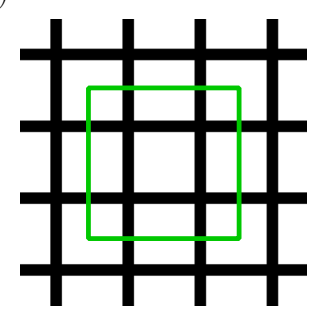

(e)

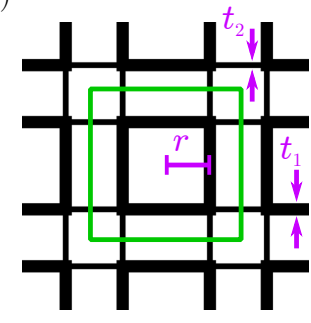

(b)

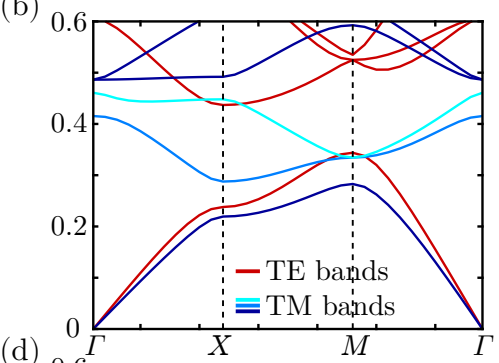

(d)

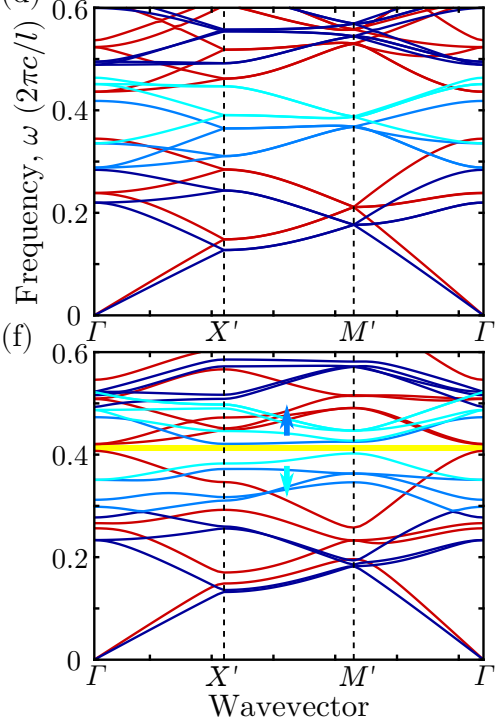

FIG. 4. (a),(c),(e) Schematics of 2D photonic crystals formed of a network of a high dielectric material with $n=3.2$ (black region) in a background of air, $n_{\text {air }}=1$. The unit cell for the corresponding band structure in (b),(d),(f) is shown as a green square. Parameters which characterize these crystals are indicated in purple. The structures in (a) and (c) are completely specified by the line thickness, $t$, while the structure in (e) is completely specified by three parameters, the thickline thickness, $t_{1}$, the thin-line thickness, $t_{2}$, and the shortest distance from a thick line to the center of its corresponding thick-line bordered square, $r$. $l$ denotes the distance between two vertices in the primitive cell. (b),(d),(f) Band structures around the border of the irreducible Brillouin Zone for the photonic crystals shown in (a),(c),(e), respectively, in which the TE bands are shown in red and the TM bands are shown in blue. In (b), the $2^{\text {nd }}$ and $3^{\text {rd }}$ TM bands are shown as light blue (medium gray) and cyan (light gray), respectively, while the corresponding folded bands in the supercell Brillouin zone in (d) and (f) are also shown in the same colors. In (b), (d), $t / l=0.32$, while in (f), $t_{1} / l=0.34, t_{2} / l=0.16$, and $r / l=0.605$, and a complete bandgap is found with width $\Delta \omega / \bar{\omega}=4.6 \%$ between the $12^{\text {th }}$ and $13^{\text {th }}$ bands (yellow rectangle). Band structures were calculated using MPB [45].

candidate primitive photonic crystal must be constructed which possesses a large bandgap for one polarization, and which is spanned by at most one or two bands in the other polarization. Second, a supercell must be generated from this primitive cell such that the degenerate contours of the folded band spanning the single-polarization bandgap lie entirely within the single-polarization bandgap. Fi-

nally, the supercell perturbation which breaks the underlying primitive cell symmetries must be designed, such that a bandgap in the degenerate contour opens before the single-polarization bandgap in the original primitive system closes.

We expect these same design principles to hold for finding complete bandgaps in three-dimensional supercell photonic crystals, but in practice we have been unable to find such a structure. Although the second and third steps in the above procedure are relatively straightforward, finding good candidate primitive cell structures is much more challenging in $3 \mathrm{D}$, as finding what would be a large bandgap spanned by only a single other band is rare. For comparison, this is relatively easy in 2D, structures with isolated dielectric elements typically possess large TM bandgaps, but not TE bandgaps, while network structures typically possess large TE bandgaps, but no TM bandgaps.

Given the extensive literature on numerical optimization of photonic crystal bandgaps [24, 29, 30, 33-35], it is perhaps surprising that the supercell crystal structures reported here have eluded discovery. As many previous studies have noted, the problem of bandgap maximization is non-convex and so optimization schemes can only yield local optima. Moreover, the search landscape of this problem appears to have sharp features, and thus requires a dense set of choices of initial parameters to ensure that an actual bandgap is found. Initializing the optimization algorithm for the supercell honeycomb lattice away from the optimized parameters, or random initializations of a pixelized unit cell obeying $C_{6 v}$ symmetry, fail to find a complete bandgap, see Appendix B.

In conclusion, we have developed a new class of photonic crystals which support complete bandgaps which stem from breaking spatial symmetries, which exhibit complete bandgaps for much lower index-contrast ratios than was previously known.

\section{Appendix A: Pixel-by-pixel nonlinear bandgap optimization}

In this appendix, we attempt to add additional degrees of freedom to the supercell honeycomb photonic crystal to see whether the bandgap of these new structures increases monotonically. To test this, we discretized our system using a set of rhomboid pixels so as to preserve $C_{6 v}$ symmetry, as shown in Fig. 5(a), and those pixels contained in, or on the boundary of, a single irreducible zone of the real-space crystal were allowed to have their dielectric values modified independently. We then used the gradient-free nonlinear optimization algorithm COBYLA [67], as implemented in NLopT [68], to optimize the dielectric values of each pixel, within the range $\left[1, \varepsilon_{h i g h}\right]$. This process revealed three different types of structures, examples of which are shown in Figs. 5(df). At low index-contrast values, the optimized pixel structure reproduced the supercell honeycomb structure, 


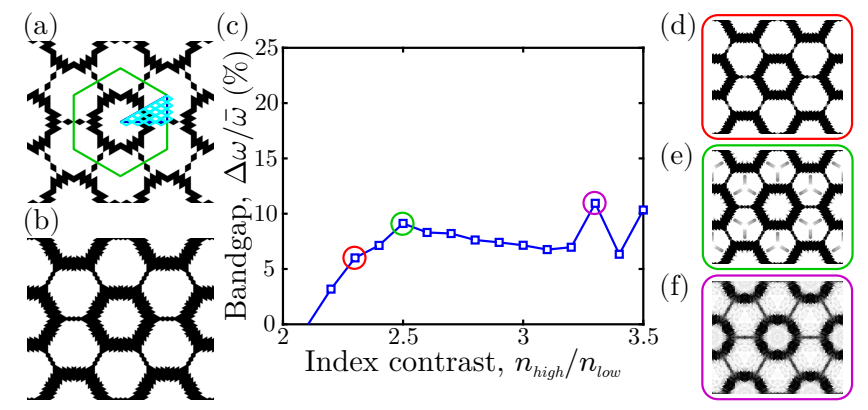

FIG. 5. (a) Schematic showing a coarse pixelized version of the supercell honeycomb photonic crystal. The unit cell is depicted in green hexagon, the irreducible spatial region is the blue triangle, and those pixels which are independent degrees of freedom are shown as cyan diamonds. (b) Initial dielectric pattern chosen for the non-linear optimization algorithm. There are 110 independent degrees of freedom in this structure. (c) Optimized bandgap as a function of index contrast for the pixelized structures. The optimization process produced three different types of structures, examples of which are shown in (d)-(f), with (d) being the structure for $n_{\text {high }} / n_{\text {low }}=2.2$, (e) $n_{\text {high }} / n_{\text {low }}=2.5$, and (f) $n_{\text {high }} / n_{\text {low }}=3.3$.

shown in Fig. 5(d), while at higher index-contrasts, additional structural features were found, which either resembled spikes, sticking out from the flat edges of the thick hexagons, Fig. 5(e), or the thick hexagon being changed to a circle, with additional holes removed from the interior, Fig. 5(f).

From these pixelized structures, we formulated four additional 'simplified' photonic crystal structures. Two of these structures had 5 independent parameters, shown in Figs. 6(b) and (c), and correspond to the optimized pixel structures observed in Figs. 5(d) and (e) respectively. Additionally, we also constructed two designs with 7 independent parameters, shown in Figs. 6(d) and (e), which correspond to adding the cutout holes in Fig. 5(c) to the design in (b), and adding the spikes in Fig. 5(b) to the design in (c), respectively. The numerically optimized complete bandgaps of these four simplified structures are then compared against the original 3-parameter supercell honeycomb crystal in Fig. 6(a). As can be seen, these structures with additional degrees of freedom do realize slightly larger complete bandgaps relative to the original supercell honeycomb photonic crystal design. None of these structures demonstrate a steady increase in their bandgap as the index contrast continues to increase, although at least one of them appears to plateau.

\section{Appendix B: Nonlinear optimization with random initialization}

In addition to the pixel-by-pixel nonlinear optimization that was performed on structures initialized with the supercell honeycomb photonic crystal, we also performed
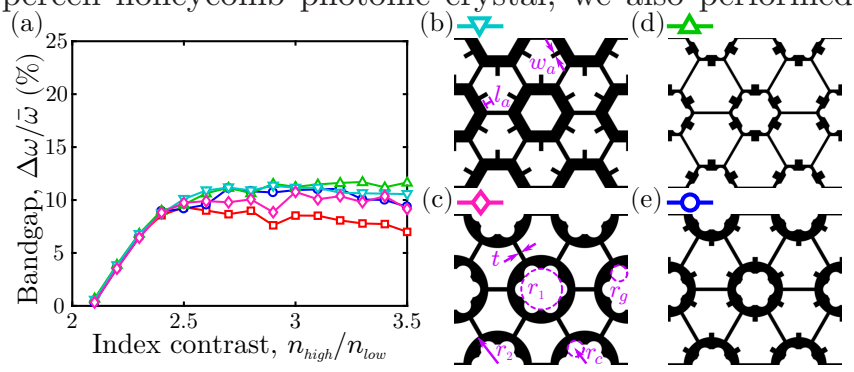

FIG. 6. (a) Numerically optimized complete photonic bandgaps as a function of index contrast for five different simplified structures depicted in (b)-(e), and the structure from Fig. 1(e). Parameters of the structures are indicated in purple.

optimizations of structures with random initializations. There were two different types of random initialization used, a first in which the dielectric of every pixel was assigned a random value between $\left[1, \varepsilon_{\text {high }}\right]$, and a second in which the dielectric of every pixel was randomly assigned either 1 or $\varepsilon_{h i g h}$, with a density of high-dielectric pixels equal to that of the pixelized supercell honeycomb structure. For both types of random initializations we ran twenty independent simulations, and none of these simulations were able to find a bandgap between the 8th and 9th bands, much less a structure which displayed a larger complete bandgap than the supercell honeycomb design.

\section{ACKNOWLEDGMENTS}

This work was supported by an AFOSR MURI program (Grant No. FA9550-12-1-0471), and an AFOSR project (Grant No. FA9550-15-1-0335).
[1] E. Yablonovitch, Phys. Rev. Lett. 58, 2059 (1987).

[2] S. John, Phys. Rev. Lett. 58, 2486 (1987).

[3] J. D. Joannopoulos, P. R. Villeneuve, and S. Fan, Nature 386, 143 (1997).

[4] J. D. Joannopoulos, S. G. Johnson, J. N. Winn, and R. D. Meade, Photonic Crystals: Molding the Flow of Light (Second Edition) (Princeton University Press,
2011).

[5] J. R. Piper and S. Fan, ACS Photonics 1, 347 (2014).

[6] J. R. Piper, V. Liu, and S. Fan, Appl. Phys. Lett. 104, 251110 (2014).

[7] S. Noda, M. Yokoyama, M. Imada, A. Chutinan, and M. Mochizuki, Science 293, 1123 (2001). 
[8] Y. Kurosaka, S. Iwahashi, Y. Liang, K. Sakai, E. Miyai, W. Kunishi, D. Ohnishi, and S. Noda, Nat Photon 4, 447 (2010).

[9] K. Hirose, Y. Liang, Y. Kurosaka, A. Watanabe, T. Sugiyama, and S. Noda, Nat Photon 8, 406 (2014).

[10] S. Fan, P. R. Villeneuve, R. D. Meade, and J. D. Joannopoulos, Appl. Phys. Lett. 65, 1466 (1994).

[11] D. Dobson and S. Cox, SIAM J. Appl. Math. 59, 2108 (1999).

[12] M. Doosje, B. J. Hoenders, and J. Knoester, J. Opt. Soc. Am. B 17, 600 (2000).

[13] S. J. Cox and D. C. Dobson, J. Comput. Phys. 158, 214 (2000).

[14] S. G. Johnson and J. D. Joannopoulos, Appl. Phys. Lett. 77, 3490 (2000).

[15] L. Shen, S. He, and S. Xiao, Phys. Rev. B 66, 165315 (2002).

[16] R. Biswas, M. Sigalas, K. Ho, and S. Lin, Phys. Rev. B 65, 205121 (2002).

[17] M. Maldovan, A. Urbas, N. Yufa, W. Carter, and E. Thomas, Phys. Rev. B 65, 165123 (2002).

[18] M. Maldovan, C. K. Ullal, W. C. Carter, and E. L. Thomas, Nat Mater 2, 664 (2003).

[19] K. Michielsen and J. S. Kole, Phys. Rev. B 68, 115107 (2003).

[20] O. Sigmund and J. S. Jensen, Philos. Trans. R. Soc. A 361, 1001 (2003).

[21] O. Toader, M. Berciu, and S. John, Phys. Rev. Lett. 90, 233901 (2003).

[22] J. S. Jensen and O. Sigmund, Appl. Phys. Lett. 84, 2022 (2004).

[23] M. B. Stanley, S. J. Osher, and E. Yablonovitch, IEICE Trans. Electron. 87, 258 (2004).

[24] C. Y. Kao, S. Osher, and E. Yablonovitch, Appl. Phys. B 81, 235 (2005).

[25] M. Maldovan and E. L. Thomas, J. Opt. Soc. Am. B 22, 466 (2005).

[26] S. Halkjær, O. Sigmund, and J. S. Jensen, Struct. Multidiscip. Optim. 32, 263 (2006).

[27] Y. Watanabe, Y. Sugimoto, N. Ikeda, N. Ozaki, A. Mizutani, Y. Takata, Y. Kitagawa, and K. Asakawa, Opt. Express 14, 9502 (2006).

[28] O. Sigmund and K. Hougaard, Phys. Rev. Lett. 100, 153904 (2008).

[29] H. Men, N. C. Nguyen, R. M. Freund, P. A. Parrilo, and J. Peraire, Journal of Computational Physics 229, 3706 (2010).

[30] A. F. Oskooi, Computation $\&$ design for nanophotonics, Thesis, Massachusetts Institute of Technology (2010).

[31] L. Jia and E. L. Thomas, Phys. Rev. A 84, 033810 (2011).

[32] X. Liang and S. G. Johnson, Opt. Express 21, 30812 (2013).

[33] H. Men, R. M. Freund, N. C. Nguyen, J. Saa-Seoane, and J. Peraire, Operations Research 62, 418 (2014).

[34] A. F. Oskooi, J. D. Joannopoulos, and S. G. Johnson, Opt. Express 17, 10082 (2009).

[35] H. Men, K. Y. K. Lee, R. M. Freund, J. Peraire, and S. G. Johnson, Opt. Express 22, 22632 (2014).

[36] M. Maldovan and E. L. Thomas, Nat Mater 3, 593 (2004).

[37] H. R. Phillip and E. A. Taft, Phys. Rev. 136, A1445 (1964).

[38] J. R. DeVore, J. Opt. Soc. Am. 41, 416 (1951).
[39] M. J. Weber, CRC Handbook of Laser Science and Technology Supplement 2: Optical Materials (CRC Press, 1994).

[40] E. Yablonovitch, J. Opt. Soc. Am. B 10, 283 (1993).

[41] S. Y. Lin, J. G. Fleming, D. L. Hetherington, B. K. Smith, R. Biswas, K. M. Ho, M. M. Sigalas, W. Zubrzycki, S. R. Kurtz, and J. Bur, Nature 394, 251 (1998).

[42] S. Noda, K. Tomoda, N. Yamamoto, and A. Chutinan, Science 289, 604 (2000).

[43] Y. A. Vlasov, X.-Z. Bo, J. C. Sturm, and D. J. Norris, Nature 414, 289 (2001).

[44] M. Qi, E. Lidorikis, P. T. Rakich, S. G. Johnson, J. D. Joannopoulos, E. P. Ippen, and H. I. Smith, Nature 429, 538 (2004).

[45] S. G. Johnson and J. D. Joannopoulos, Opt. Express 8, 173 (2001).

[46] A. Cerjan, A. Raman, and S. Fan, Phys. Rev. Lett. 116, 203902 (2016).

[47] T. Siefke, S. Kroker, K. Pfeiffer, O. Puffky, K. Dietrich, D. Franta, I. Ohldal, A. Szeghalmi, E.-B. Kley, and A. Tnnermann, Advanced Optical Materials 4, 1780 (2016).

[48] J. Ballato, T. Hawkins, P. Foy, R. Stolen, B. Kokuoz, M. Ellison, C. McMillen, J. Reppert, A. M. Rao, M. Daw, S. Sharma, R. Shori, O. Stafsudd, R. R. Rice, and D. R. Powers, Opt. Express 16, 18675 (2008).

[49] F. Yaman, H. Pang, X. Xie, P. LiKamWa, and G. Li, in Conference on Lasers and Electro-Optics/International Quantum Electronics Conference (2009), paper CTuDD7 (Optical Society of America, 2009) p. CTuDD7.

[50] J. C. Knight, T. A. Birks, P. S. J. Russell, and D. M. Atkin, Opt. Lett. 21, 1547 (1996).

[51] J. C. Knight, J. Broeng, T. A. Birks, and P. S. J. Russell, Science 282, 1476 (1998).

[52] S. E. Barkou, J. Broeng, and A. Bjarklev, Opt. Lett. 24, 46 (1999).

[53] P. Russell, Science 299, 358 (2003).

[54] M. Soljačić, S. G. Johnson, S. Fan, M. Ibanescu, E. Ippen, and J. D. Joannopoulos, J. Opt. Soc. Am. B 19, 2052 (2002).

[55] F. Benabid, J. C. Knight, G. Antonopoulos, and P. S. J. Russell, Science 298, 399 (2002).

[56] J. F. McMillan, X. Yang, N. C. Panoiu, R. M. Osgood, and C. W. Wong, Opt. Lett. 31, 1235 (2006).

[57] Y. L. Hoo, W. Jin, C. Shi, H. L. Ho, D. N. Wang, and S. C. Ruan, Appl. Opt. 42, 3509 (2003).

[58] T. Ritari, J. Tuominen, H. Ludvigsen, J. C. Petersen, T. Srensen, T. P. Hansen, and H. R. Simonsen, Opt. Express 12, 4080 (2004).

[59] J. B. Jensen, L. H. Pedersen, P. E. Hoiby, L. B. Nielsen, T. P. Hansen, J. R. Folkenberg, J. Riishede, D. Noordegraaf, K. Nielsen, A. Carlsen, and A. Bjarklev, Opt. Lett. 29, 1974 (2004).

[60] S. O. Konorov, A. M. Zheltikov, and M. Scalora, Opt. Express 13, 3454 (2005).

[61] V. R. Manfrinato, L. Zhang, D. Su, H. Duan, R. G. Hobbs, E. A. Stach, and K. K. Berggren, Nano Lett. 13, 1555 (2013).

[62] W. Park, J. Rhie, N. Y. Kim, S. Hong, and D.-S. Kim, Sci. Rep. 6, 23823 (2016).

[63] I. Aharonovich, A. D. Greentree, and S. Prawer, Nat. Photon. 5, 397 (2011).

[64] B. J. M. Hausmann, I. Bulu, V. Venkataraman, P. Deotare, and M. Lončar, Nat. Photon. 8, 369 (2014). 
[65] M. J. Burek, C. Meuwly, R. E. Evans, M. K. Bhaskar, A. Sipahigil, S. Meesala, D. D. Sukachev, C. T. Nguyen, J. L. Pacheco, E. Bielejec, M. D. Lukin, and M. Lončar, arXiv:1612.05285 (2016).

[66] S. G. Johnson, S. Fan, P. R. Villeneuve, J. D. Joannopoulos, and L. A. Kolodziejski, Phys. Rev. B 60, 5751
(1999).

[67] M. J. D. Powell, in Advances in Optimization and Numerical Analysis, Mathematics and Its Applications (Springer, Dordrecht, 1994) pp. 51-67, dOI: 10.1007/97894-015-8330-5_4.

[68] S. G. Johnson, "The nlopt nonlinear-optimization package," Http://ab-initio.mit.edu/wiki/index.php/NLopt. 